Bundesgesundheitsbl 2017 ·60:130-140

DOI 10.1007/s00103-016-2490-9

Online publiziert: . undefined

(c) Springer-Verlag Berlin Heidelberg 2016
Stellungnahme der Kommission Umweltmedizin, einer Kommission des Robert

Koch-Instituts (RKI) und des Umweltbundesamtes (UBA)

\section{Gesundheitliche Bewertung von Maßnahmen und Energieträgern im Rahmen der Energiewende aus umweltmedizinischer Sicht}

\section{Energiewende - quo vadis?}

Die Bundesregierung forciert die Energiewende für eine langfristige sowie nachhaltige Entwicklung und Sicherstellung der Energieversorgung [1]. Die Einführung neuer Technologien zur Energiegewinnung und Energieeinsparung wird dabei z.B. mit dem Erneuerbare-Energien-Gesetz (EEG; [45]) für den Ausbau der erneuerbaren Energien im Strommarkt oder zinsverbilligten Krediten der Kreditanstalt für Wiederaufbau für die Wärmebereitstellung aus erneuerbarer Energie oder die Sanierung von Gebäuden gefördert. Obwohl die neu eingeführten Technologien die wesentlichen gesundheitlichen Risiken des bestehenden Energieversorgungssystems nicht aufweisen, ist die kritische Betrachtung und Bewertung möglicher Auswirkungen sowohl auf die Gesundheit der Menschen als auch auf die Umwelt und das Ökosystem aus umweltmedizinischer Sicht trotz zum Teil flächendeckender Einführung nicht ausreichend. Daher soll die vorliegende Stellungnahme einen Beitrag zu dieser sehr komplexen Thematik leisten und den aktuellen Stand zu umweltmedizinischen Aspekten der Energiewende diskutieren, dabei aber nicht nur auf die direkten Wirkungen der neuen Technologien, sondern auch auf die zum Teil indirekten Auswirkungen, wie beispielsweise die mögliche Änderung der Innenluft/des Innenraumklimas bei wärmedämmenden Maßnahmen, eingehen. Abschließende Empfehlungen sollten Gegenstand einer interdisziplinären Be- trachtung unter Berücksichtigung weiterer Expertisen aus Technik, Ingenieurswissenschaften etc. sein, sind aber nicht das Ziel der vorliegenden Stellungnahme.

\section{Veränderungen durch die Energiewende}

Unter den Begriff der Energiewende fallen unterschiedliche Maßnahmen. Konkret zählen dazu unter anderem der Ausstieg aus der Kernenergie, die Reduktion der Energiegewinnung aus fossilen Brennstoffen (Braun- und Steinkohle, Erdgas und -öl), der Ausbau von Windenergie und Photovoltaik, die zum Teil notwendige Modernisierung der Stromnetze, die Förderung der energetischen Gebäudesanierung, aber auch der Einsatz von energieeffizienten Geräten, wie z.B. Energiesparlampen. Diese Maßnahmen sollen dazu beitragen, die Entstehung von Treibhausgasen und anderen Luftschadstoffen zu vermeiden.

Durch die große Tragweite der Projekte, die mit der Energiewende einhergehen, zählt sie zu einer der größten umweltpolitischen Herausforderungen. Sie erfordert in ihrer Umsetzung daher unweigerlich Kompromisse. Bei der Entscheidungsfindung muss zwischen Ökologie und Wirtschaftlichkeit, sozialer Gerechtigkeit und internationaler Wettbewerbsfähigkeit und insbesondere auch zwischen europäischen Perspektiven und lokalen Bürgerinteressen abgewogen werden.

Grundsätzlich ist festzuhalten, dass der Rückbau der klassischen Energiege- winnung aus der Verbrennung fossiler Brennstoffe, wie Braun- und Steinkohle, einen erheblichen Beitrag zur Vermeidung der Freisetzung von klima- und gesundheitsgefährdenden Stoffen wie z.B. Kohlenstoffdioxid, Feinstaub, Stickoxiden, Schwefeldioxid und Schwermetallen leisten kann.

Die Reduktion der Kohleverstromung führt zur Verringerung der Emissionen von dafür typischen Luftschadstoffen (z. B. $\mathrm{NO}_{\mathrm{x}}$, Feinstaub), die in einem direkten Zusammenhang mit negativen Gesundheitseffekten (z. B. Lungenkrebs, Herzkreislauferkrankungen) stehen [2], was eine gewisse Reduktion der diesbezüglichen Krankheitslast erwarten lässt. Erste Untersuchungen für Deutschland zeigen, dass auf Feinstaub, der aus den Kohlekraftwerken emittiert wird, ca. 2700-3100 vorzeitige Sterbefälle bzw. 29.000-33.000 verlorene Lebensjahre pro Jahr zurückzuführen sind [3, 4].

Durch den Ausstieg aus der Kernenergiewirtschaft werden langfristig keine neuen nuklearen Abfälle anfallen. Gesundheitliche Gefahren können jedoch weiterhin durch den Transport sowie die Endlagerung nuklearen Materials, auftretende Havarien und daraus resultierender Strahlung, sowie die mögliche Kontamination des Grundwassers entstehen.

Bei einer Bilanzierung aus umweltmedizinischer Sicht sind auch diese nach heutigem Stand verminderten, aber weiterhin bestehenden Gesundheitsrisiken der alten Energieträger zu berücksichtigen. Inwieweit die neuen und veränder- 
ten Maßnahmen Auswirkungen auf die Gesundheit haben, ist bislang noch nicht ausreichend betrachtet und wissenschaftlich untersucht worden. Der derzeitige Forschungsbedarf wird für die jeweiligen Energieträger/Energieeinsparungsmaßnahmen unter „Kenntnislücken zu gesundheitlichen Aspekten“ aufgeführt.

\section{Diskussion umweltmedizini- scher Komponenten sowie Darstellung von Lücken im aktuellen Kenntnisstand zu den einzelnen Energieträgern oder Maßnahmen}

Die mit der Energiewende verbundenen weitreichenden technologischen und strukturellen Änderungen werden aller Voraussicht nach in unterschiedlichen gesellschaftlichen Bereichen Auswirkungen zeigen. Bereits vielfach untersucht wurden die ökonomischen und umweltbezogenen positiven und negativen Auswirkungen der Energiewende mit Fokus auf den Bereichen erneuerbare Energien (Strom, Wärme), Verkehr, Mobilität, Bauen und Recycling.

Da die energiepolitischen Maßnahmen das Schutzgut menschliche Gesundheit in erheblichem Maße betreffen können, gebietet es der vorsorgende Gesundheitsschutz, die Chancen und Risiken für die menschliche Gesundheit, die mit den energiepolitischen Entwicklungen und Neuerungen entstehen könnten, frühzeitig zu identifizieren und diskutieren und diesen ggf. entgegenzuwirken.

Im Folgenden werden umweltmedizinische Aspekte ausgewählter Maßnahmen und Energieträger, die im Rahmen der Energiewende von Bedeutung sind, kritisch betrachtet. Der Fokus der vorliegenden Stellungnahme liegt dabei auf umweltmedizinischen Aspekten: In erster Linie sollen hier die direkten Auswirkungen auf die Gesundheit betrachtet und aufgezeigt, aber auch die im Sinne einer Gesamtbilanzierung zwingend zu beachtenden indirekten Auswirkungen diskutiert werden.

Dabei gilt es, nicht nur die verschiedenen Technologien der Energiegewinnung, sondern auch die Maßnahmen, die zur Energieeinsparung eingesetzt werden, im Hinblick auf die potentielle Gefährdung des Schutzguts menschliche Gesundheit kritisch zu analysieren und Vergleiche zwischen den unterschiedlichen Alternativen, die im Kontext der Energiewende diskutiert werden, anzustellen.

\section{Windenergieanlagen}

Windenergieanlagen nutzen Wind als eine erneuerbare Energiequelle. Hierbei wird die kinetische Energie von Wind, d. h. die bewegten Luftmassen der Atmosphäre technisch genutzt. Die Windenergie wird seit Langem genutzt, zu Beginn vor allem mit Windmühlen oder Segelschiffen, heute steht vor allem die Stromerzeugung durch Windenergieanlagen (WEA) im Vordergrund.

Die WEA nehmen in den Strategien zur Energiewende eine Schlüsselrolle ein, denn schon im Jahr 2014 lag der Anteil der Bruttostromerzeugung durch WEA an Land bei ca. 9,5\% [5]. Zusammen mit Sonnenenergie aus Photovoltaik soll die Windenergie in der Zukunft die wichtigste Quelle für die Energiegewinnung in Deutschland werden.

Kenntnisstand zu direkten gesundheitlichen Auswirkungen: Seit Beginn der Errichtung von WEA wurden einige potentiell gesundheitsgefährdende Eigenschaften der WEA diskutiert, wobei einige der Probleme, wie periodischer Schattenwurf, Lichteffekte an den Reflexionsflächen („Diskoeffekt“ bzw. „Stroboskopeffekt“) oder Eiswurf durch die technische Modernisierung und Anpassung bereits beseitigt bzw. stark reduziert werden konnten. So sorgen z. B. technische Regelungen dafür, dass bei Schattenwurfkonditionen der tatsächliche Schattenwurf auf $30 \mathrm{~min} / \mathrm{Tag}$ (8 Stunden pro Jahr) reduziert wird. Eine matte Lackierung der WEA und beheizte Rotorblätter helfen, dass Lichtreflexionen ausbleiben und wirken bei Bedarf der Eisbildung an den Rotorblättern entgegen. Studien zeigten, dass Belästigungen auch durch die Warnkennzeichnung von WEA (mit einer Höhe von mehr als 100 m) verursacht werden können [6]. Auch hier wurde und wird an technischen Lösungen zur Reduktion der Belästigung, unter Beibehaltung der Sicherheit für den Flugverkehr, gearbeitet. Eine vorgeschriebene Synchronisierung der Warnbeleuchtung in Windparks, und die derzeit erprob- te bedarfsgerechte Warnbeleuchtung bei Näherung von Luftfahrzeugen (z.B. über Radarsysteme) können entsprechend die Belästigung reduzieren.

Neben diesen weitestgehend geklärten Problemen werden zurzeit Gesundheitsrisiken wie lärmassoziierte Aspekte sehr ausführlich diskutiert. Grundsätzlich ist zwischen Schall im Hörbereich, tieffrequentem Schall $(<100 \mathrm{~Hz})$ sowie Infraschall $(<20 \mathrm{~Hz}) \mathrm{zu}$ unterscheiden.

Menschen nehmen Schall primär über das Ohr wahr. Diese akustische Wahrnehmung wird als "Hören“ bezeichnet. Im Bereich tiefer Frequenzen (unterhalb 100 Hertz) nimmt jedoch die Empfindung der Tonhöhen ab. Auch im Infraschallbereich ist eine Art „Hören“ technisch möglich: Hierfür sind jedoch deutlich höhere Schalldruckpegel notwendig als beim Hörschall. Das Gehör ist auch im Infraschallbereich das sensibelste Sinnesorgan des Menschen [7]. Erst wenn die Schalldruckpegel deutlich über der Hörschwelle liegen, kann der tieffrequente Schall auch mit dem Tastsinn (taktil) und dem Gleichgewichtssinn (vestibulär) wahrgenommen werden. Die Hörschwelle gibt an, wie laut ein Ton sein muss, damit er vom menschlichen Gehör wahrgenommen werden kann. Sie ist von Mensch zu Mensch natürlicherweise unterschiedlich. Daher wird als statistischer Wert der sogenannte Median herangezogen: Bei diesem Wert kann die Hälfte der Bevölkerung den frequenzspezifischen Ton bei dem angegebenen Schalldruckpegel nicht hören, die anderen $50 \%$ aber schon [8].

Beim Infraschall gibt es hier eine Besonderheit: Die Unterschiede in der individuellen Hörschwelle sind im Infraschallbereich stärker ausgeprägt als im Hörschallbereich. Es wird davon ausgegangen, dass die individuelle Hörschwelle bei etwa zwei Drittel der Bevölkerung in einem Bereich von plus/minus sechs Dezibel $(\mathrm{dB})$ um die in $\bullet$ Tab. 1 angegebenen Werte liegt. Um den stärkeren individuellen Unterschieden gerecht zu werden, wurde in neueren Regelwerken die sogenannte Wahrnehmungsschwelle benannt, die durch einen anderen statistischen Wert definiert ist (90-Prozent-Perzentil der Hörschwellenverteilung): Die Wahrnehmungsschwelle entspricht demnach einem Schalldruckpegel, bei dem $90 \%$ der 
Tab. 1 Hörschwellen ${ }^{1}$ und Wahrnehmungsschwellen ${ }^{2}$ im Infraschall-Frequenzbereich

\begin{tabular}{|c|c|c|c|c|c|}
\hline \multirow[t]{2}{*}{ Schwelle } & \multicolumn{5}{|c|}{ Schalldruckpegel $[\mathrm{dB}(\mathrm{Z})]^{3}$ bei einer Frequen $\mathrm{z}^{4}$ von } \\
\hline & $\mathrm{Hz}$ & $10 \mathrm{~Hz}$ & $12,5 \mathrm{~Hz}$ & $16 \mathrm{~Hz}$ & $20 \mathrm{~Hz}$ \\
\hline Hörschwelle & 103 & 95 & 87 & 79 & 71 \\
\hline Wahrnehmungsschwelle & 100 & 92 & 84 & 76 & 68,5 \\
\hline \multicolumn{6}{|c|}{$\begin{array}{l}\text { DIN 45680, März 1997: Messung und Bewertung tieffrequenter Geräuschimmissionen in der Nachbarschaft } \\
\text { Entwurf DIN 45680, September 2013: Messung und Bewertung tieffrequenter Geräuschimmissionen } \\
\text { dB(Z) unbewerteter mittlerer Schalldruckpegel } \\
\text { Terzmitten-Frequenz } \\
\text { (Quelle: Bayerisches Landesamt für Umwelt; 2014) }\end{array}$} \\
\hline
\end{tabular}

Bevölkerung den Ton nicht mehr hören kann. Daraus ergibt sich auch, dass $10 \%$ der Bevölkerung besser hören, also den Ton auch bei dem angegebenen Schalldruckpegel noch hören können. Außerdem zeigt $\bullet$ Tab. 1 noch einen weiteren wichtigen Aspekt: Je tiefer die Frequenz ist, desto höher muss der Schalldruckpegel - also die Lautstärke - sein, damit der Mensch etwas wahrnimmt. So muss bei 8 Hertz der Schalldruckpegel bei 100 Dezibel liegen, bei 16 Hertz hingegen genügen 76 Dezibel. Bei 100 Hertz würden sogar 23 Dezibel ausreichen [8].

Für den Bau und Betrieb von WEA müssen jeweils die Immissionsrichtwerte (IR) nach der Technischen Anleitung zum Schutz gegen Lärm (TA-Lärm) eingehalten werden, die für die jeweiligen Siedlungsgebiete gelten. Eine pauschale Bemessung der Abstände wäre hierbei nur wenig zielführend, weil bei der Betrachtung des von der WEA ausgehenden Lärms auch die bereits vorhandene Lärmvorbelastung (z. B. durch Straßenverkehr oder Schienenverkehr) mit berücksichtigt werden soll und jede Entscheidung entsprechend individuell getroffen werden muss. Es ist somit bei jeder Errichtung von WEA eine Einzelfallprüfung notwendig, um die im Regelwerk TA-Lärm definierten Immissionsrichtwerte einzuhalten. Diese variieren je nach Siedlungstyp (siehe • Tab. 2).

Es ist durchaus kritisch anzumerken, dass eine Bewertung nach $\mathrm{dB}(\mathrm{A})$ den tieffrequenten Anteil $(<100 \mathrm{~Hz})$ unterschätzt, zudem basieren die Werte der Technische Anleitung Lärm (TA-Lärm) nicht auf bevölkerungsbasierten dosis-wirkungsbezogenen Studien.

Die Einhaltung der Immissionsrichtwerte nach TA-Lärm ist die Voraussetzung für den Bau und Betrieb von
WEA. Neben der tatsächlich gemessenen Schalldruckpegelhöhe wird vermehrt die Problematik der sog. Amplitudenmodulation diskutiert. Diese erzeugt Schall im hörbaren Bereich, es handelt sich hierbei um eine Veränderung der Lautstärke (innerhalb der Grenzwerte), die eine erhöhte Aufmerksamkeit auf sich zieht und dadurch zur Belästigung und zu Schlafstörungen führen kann. Inwieweit dies der jeweiligen Konstruktion der Anlagen geschuldet ist, ist bislang noch offen.

Viel diskutiert ist die Frage der Emission von Infraschall durch WEA. Die Tatsache, dass WEA (wie auch andere technische Infrastrukturen des Alltags) Infraschall produzieren, ist zunächst richtig, jedoch zeigen aktuelle Studien aus Baden-Württemberg und Bayern, dass der durch WEA erzeugte Infraschall deutlich unterhalb der menschlichen Hör- bzw. Wahrnehmungsschwelle nach DIN 45680 liegt $[8,9]$. In der Studie der Landesanstalt für Umwelt, Messungen und Naturschutz Baden-Württemberg (LUBW) wurden Messungen für unterschiedliche Anlagentypen und Abstände zu den Anlagen durchgeführt. Hierbei zeigt sich, dass bereits ab einer Entfernung von 300 Metern die Infraschallemissionen zu einem großen Teil auf die durch den Wind erzeugten Geräusche zurückzuführen sind. Ein signifikanter Unterschied der Lärmemissionen zwischen einer Anlage in Betrieb und einer Anlage außer Betrieb konnte ebenfalls nicht festgestellt werden [9]. Nach derzeitiger Studienlage kann davon ausgegangen werden, dass WEA zwar Infraschall emittieren, die gemessenen Werte jedoch unterhalb der Wahrnehmungsschwelle liegen und der gemessene Schall ab einer gewissen Entfernung (Beispiel s.o.) zur WEA eher auf andere Quellen (z.B. Wind, Verkehr) zurückzuführen ist.

Gemessen an den Beschwerden, die durch betroffene Bürgerinnen und Bürger an die deutschen Landesämter herangetragen wurden, dominieren in Bezug auf Infraschall und tieffrequenten Schall andere Lärmquellen, wie z.B. Wärmepumpen $(9,3 \%)$, Biogasanlagen $(8,4 \%)$ und Blockheizkraftwerke (6,5\%). Für den Bereich der Windkraftanlagen liegt die Beschwerderate deutlich niedriger $(3,3 \%)$ [10]. Hierbei sollte allerdings beachtet werden, dass die Beschwerden, die von den Personen an die Landesämter herangetragen worden sind, nicht zwingend auf Belastungen durch z. B. WEA zurückgeführt werden müssen. Ohne eine tatsächliche Belastungsprüfung (Messung von Infraschall und Überprüfung der Quellen) kann eine eindeutige Zuordnung der Belastung alleinig beruhend auf selbstberichteten Aussagen der Bürgerinnen und Bürger nicht erfolgen.

Anders sieht es für die Schallimmission im Bereich von $20 \mathrm{~Hz}$ bis $100 \mathrm{Herz}$ aus. Dieser tieffrequente Anteil des Frequenzspektrums, oberhalb der Hör- und Wahrnehmungsschwelle, kann unter Umständen eine wichtige Rolle in der Lärmbelästigung der Anwohner und Anwohnerinnen spielen [7].

Kenntnislücken zu gesundheitlichen Aspekten: Insgesamt ist festzuhalten, dass die Effekte von Infraschall und tieffrequentem Schall auch unterhalb der Wahrnehmungsschwelle noch nicht ausreichend und abschließend erforscht wurden [11].

Aspekte zu den indirekten Auswirkungen: Die WEA zeichnen sich vor allem dadurch aus, dass während des Betriebs keine klimagefährdenden Gase entstehen und die Flächeninanspruchnahme im Vergleich zu anderen Energieerzeugungsmethoden deutlich geringer ist. Allerdings führen diese Anlagen zu landschaftlichen Veränderungen, z. B. Beeinträchtigung der landschaftlichen Integrität kulturlandschaftsprägender Denkmale und haben auch Auswirkungen auf den Tourismus [12]. Auch die Hinderniskennzeichnung kann sich belästigend auswirken. Die Präsenz und Dichte von WEA in einigen Teilen Deutschlands hat auch zu umweltpsychologischen Wirkungen geführt, wie etwa der Belästigung durch vi- 
Tab. 2 Einzuhaltende Immissionsrichtwerte (dB(A) nach Siedlungstyp (TA-Lärm; BMI, 1998)

\begin{tabular}{lll}
\hline Gebietsart & Tag & Nacht \\
\hline Industriegebiete & $70 \mathrm{~dB}(\mathrm{~A})$ & $70 \mathrm{~dB}(\mathrm{~A})$ \\
Gerwerbegebiete & $65 \mathrm{~dB}(\mathrm{~A})$ & $50 \mathrm{~dB}(\mathrm{~A})$ \\
Kern-, Dorf-, Mischgebiete & $60 \mathrm{~dB}(\mathrm{~A})$ & $45 \mathrm{~dB}(\mathrm{~A})$ \\
Allgemeine Wohngebiete & $55 \mathrm{~dB}(\mathrm{~A})$ & $40 \mathrm{~dB}(\mathrm{~A})$ \\
Reine Wohngebiete & $50 \mathrm{~dB}(\mathrm{~A})$ & $35 \mathrm{~dB}(\mathrm{~A})$ \\
Kurgebiete, Krankenhäuser, Pflegeanstalten & $45 \mathrm{~dB}(\mathrm{~A})$ & $35 \mathrm{~dB}(\mathrm{~A})$
\end{tabular}

suelle Merkmale und einer persönlichen negativen Einstellung gegenüber WEA.

\section{Biogas}

In Biogas-Anlagen werden überwiegend nachwachsende Rohstoffe wie Raps, Hirse, Grassilage und in Deutschland häufig Mais als Substrat eingesetzt. Die Rohstoffe werden durch spezielle Bakterien unter Ausschluss von Sauerstoff (anaerob) in vier Phasen abgebaut. Die dabei von den Bakterien produzierten Gase bestehen zu zwei Dritteln aus Methan, daneben aus Kohlendioxid, Sauerstoff, Stickstoff und einer geringen Menge weiterer Gase. In Blockheizkraftwerken wird das durch die Gärung entstandene Biogas für die Stromund Wärmeerzeugung genutzt.

Kenntnisstand $z u$ direkten gesundheitlichen Auswirkungen: Im BMBF Forschungsvorhaben „Risiken durch Abwässer aus der intensiven Tierhaltung für Grund- und Oberflächenwasser" wurden folgende Auswirkungen von Biogasanlagen identifiziert [47] [Aus „Verbundprojekt RiskAGuA: Risiken durch Abwässer aus der intensiven Tierhaltung für Grund und Oberflächenwasser“, BMBF Forschungsvorhaben, Förderkennzeichen 02WRS1274A (2012-2015) siehe Homepage http://www.riskagua.de]:

- Schwermetalle (z. B. Kupfer, Zink) werden durch Biogasanlagen nicht zurückgehalten [13].

- Im Gärrest konnte eine deutliche Zunahme von Bakterien der klinisch unbedenklichen Familie Bacillaceae nachgewiesen werden.

- Der überwiegende Anteil der Bakterien im Gärrest besteht aus nicht kultivierbaren Methanbakterien (Domäne: Archaea).

- Kultivierungsunabhängige Analysen belegen, dass bisher nicht beschrie- bene Vertreter des Phylum Firmicutes den größten Teil der Bakterien in Gülle/Festmist und Gärrest ausmachen.

- Resistenzgene und resistente Bakterien werden über die Passage in der Biogasanlage reduziert, das bedeutet eine Abnahme des ökotoxikologischen Potentials.

- Die Resistenzmuster der Isolate aus Gülle/Festmist und Gärrest unterscheiden sich nur geringfügig.

- Quantitative Analysen zeigen eine positive Korrelation zwischen Antibiotikaeinsatz und Resistenzgenen.

- Die Zahl der Resistenzgen-Kopien in Gärresten ist gegenüber Gülle/Festmist reduziert.

- Der qualitative und quantitative Nachweis von Resistenzgenen lässt keine direkte Aussage über das Vorhandensein pathogener Bakterien, die eine Resistenz ausprägen, zu.

- Einige Veterinärantibiotika (Tetracycline, Sulfonamide) werden durch Biogasanlagen weitgehend eliminiert.

- Bakterien: Klinisch relevante Mikroorganismen wie Salmonellen, Clostridium botulinum, C. difficile und Staphylococcus aureus wurden in Gülle/ Festmist und Gärrest nicht nachgewiesen.

- Biogasanlagen reduzieren den Anteil der kultivierbaren, potentiell pathogenen Bakterien der Gattungen Escherichia, Shigella, Enterococcus, Staphylococcus.

- Durch (Über)düngung auf den Anbauflächen der in Biogasanlagen verwendeten Substrate wie z. B. Mais, können Grundwasserverunreinigungen hervorgerufen werden.

- Hochentzündliche und toxisch wirkende Gase (z. B. Methan und Schwefelwasserstoff) können entweichen.
- In der Umgebung können Geruchsbelästigungen auftreten.

- Das Verbrennen von Biomasse stellt in der EU eine wichtige Quelle der Luftverschmutzung dar [14].

Kenntnislücken zu gesundheitlichen Aspekten: Beim bestimmungsgemäßen Betrieb der Biogasanlage werden humanpathogene Bakterien (z. B. Clostridium botulinum, Clostridium difficile) oder antibiotikaresistente Erreger wie Methicillin-resistenter Staphylococcus aureus (MRSA) und Bakterien, hier insbesondere Escherichia Coli, die Enzyme mit einem breiten Spektrum (Extended Spectrum Beta-Lactamases/ ESBL) produzieren, welche viele Antibiotika unwirksam machen, wie z. B. ESBLE. coli im Gärprozess reduziert. Über die Auswirkungen insbesondere bei Störungen oder bei nicht bestimmungsgemäßem Betrieb, wie beispielsweise beim Einsatz kontaminierter Bioabfälle, ist wenig bekannt. Sollte es zu einer Vermehrung während des Gärprozesses kommen, erfolgt durch das Ausbringen der Gärreste auf die Felder ein potenzieller Eintrag solcher Erreger in die Nahrungskette. Erkenntnislücken gibt es zur Verbreitung der austretenden Stoffe - Gase, Partikel, Bakterien etc. und der dadurch im weiteren Umfeld einer Biogasanlage auftretenden Belastungen, so dass eine umweltmedizinische Bewertung zum aktuellen Zeitpunkt nicht abschließend möglich ist. Aus umweltmedizinischer Sicht fehlen sowohl Luftmessdaten als auch Daten zu gesundheitlichen Auswirkungen. Inwieweit auch Geruchs Belastungen gesundheitlich $\mathrm{zu}$ bewerten sind, wird zunehmend diskutiert [15]. Aus Sicht des Katastrophenschutzes sind Störfälle in Biogasanlagen in der Störfallverordnung noch nicht hinreichend abgebildet, auch hieraus ergibt sich aus umweltmedizinischer Sicht ein Risiko.

Aspekte zu den indirekten Auswirkungen: Beim Betrieb von Biogasanlagen können klimarelevante Gase emittiert werden, es kann zu Stickstoff-Auswaschungen kommen und es besteht die Gefahr der Grundwasserkontaminierung mit Nitrat aus den Gärresten.

Biogasanlagen hatten nur relative kurze Zeit das Image eines vielversprechenden neuen Hoffnungsträgers im Bereich der erneuerbaren Energien. Wenngleich es 
sich bei den Rohstoffen, die in Biogasanlagen verwendet werden, um nachwachsende Rohstoffe handelt, ist die Energieerzeugung in Biogasanlagen nach Einbezug der gesamten Produktionskette nicht treibhausgasneutral und der Flächenverbrauch ist im Vergleich zu Windenergieoder Photovoltaikanlagen wesentlich höher. Diese Technologie birgt bei Unfällen und Havarien zudem Gefahren für Menschen und Rettungskräfte. Es ist daher umso wichtiger, Rettungsdienste und Feuerwehren für die potenziellen Risiken zu sensibilisieren, um Sicherheitsstrategien $\mathrm{zu}$ etablieren und Biogasanlagen auch im Rahmen der Gefahrenabwehr in die Gefahrenabwehrpläne aufzunehmen. Viele Anlagen befinden sich im ländlichen Raum und damit oft im Zuständigkeitsbereich kleiner Wehren. Diese müssen mit geeignetem Messgerät und Schutzbekleidung ausgerüstet und entsprechend ausund fortgebildet werden [16].

Das Erneuerbare-Energien-Gesetz (EEG; [45]) förderte zunächst den Einsatz von Biogasanlagen, jedoch wurden bereits im EEG 2014 Subventionierungen für Biogasanlagen reduziert und Einschränkungen bestimmt, denen zufolge nur noch Rest- und Abfallprodukte in Biogasanlagen verwertet werden sollen, um dem intensiven Anbau von nachwachsenden Rohstoffen, das heißt dem Flächenverbrauch, der Überdüngung und der Monokultur entgegen zu wirken. Dies geschah vor dem Hintergrund, dass durch den intensiven Einsatz von Biogasanlagen wesentliche Klima-, Natur-, Umwelt- und Gesundheitsschutzziele als gefährdet eingeschätzt wurden.

\section{Holzfeuerung}

Die Nutzung von privaten Kleinfeuerungsanlagen ist bei der Verbrennung von nachhaltig angebautem $\mathrm{Holz}$ eine $\mathrm{CO}_{2}$-neutrale Wärmegewinnungsmethode. Dies kann allerdings bei vielen käuflich erwerbbaren Materialien nicht garantiert werden. Besonders bedenklich ist hierbei der Ausstoß diverser Luftschadstoffe und Feinstäube, die unter anderem auf inadäquates Brennmaterial zurückzuführen sind. Die Gesamtmenge des Feinstaubs aus Kaminöfen und anderen Holzfeuerungsanlagen in Deutschland übersteigt mittlerweile diejenige aus den Auspuffrohren aller Pkw, Lkw und Motorräder [17]. Wie viel Feinstaub ein Ofen tatsächlich ausstößt, hängt aber nicht nur von Art und Alter der Anlage ab. Auch die Art der Befeuerung, der Wartungszustand der Anlagen sowie die Auswahl und Qualität des genutzten Holzes spielen eine Rolle.

Die novellierte Bundesimmissionsschutzverordnung für mittlere und kleine Feuerungsanlagen (1.BImSchV; [43]) regelt im Sinne der Luftreinhaltung die Anforderungen an das Emissionsverhalten, auch von Pelletkesseln und -öfen. Für den Feinstaubausstoß sind die Grenzwerte $a b$ dem 1.1.2015 für neu in Betrieb genommene Anlagen (für ältere Anlagen gelten Übergangsfristen) verpflichtend einzuhalten (Staub für Pelletkessel 0,02 g/m ${ }^{3}$-; für Pelletofen luftgeführt $0,03 \mathrm{~g} / \mathrm{m}^{3}$-; für Pelletofen wassergeführt $0,02 \mathrm{~g} / \mathrm{m}^{3}$ ). Durch die große Verbreitung von Kleinfeuerungs- und Holzfeuerungsanlagen im ländlichen wie im urbanen Raum ist die Feinstaubbelastung durch Holzfeuerung entsprechend weit verbreitet.

Kenntnisstand zu direkten gesundheitlichen Auswirkungen: Die Kleinfeuerungsanlagen produzieren Feinstaub mit Partikeln, die auch in die Lunge eindringen können. Feinstaub (engl. particulate matter; PM) - wird in unterschiedliche Klassen (Fraktionen) eingeteilt. Maßeinheit ist die Partikelgröße. $\mathrm{PM}_{10}$ ist die Kategorie für Teilchen, die kleiner sind als zehn Mikrometer. Die Kategorie $\mathrm{PM}_{2,5}$ umfasst entsprechend Teilchen, die kleiner sind als 2,5 Mikrometer, und tiefer in Atemwege und Lunge sowie leichter in den Organismus gelangen können. Mehr als $90 \%$ des Staubanteils von kleinen Holzfeuerungsanlagen wird durch Staub der Partikelgröße $\mathrm{PM}_{10}$ also von inhalierbaren Staubteilchen bestimmt.

Die Belastung mit Feinstaub $\left(\mathrm{PM}_{2.5}\right)$ kann sowohl akut gesundheitliche Beschwerden z. B. akute Reizwirkung auf die Atemwege und bei Personen mit bestehenden Asthmaerkrankungen Exazerbationen verursachen, aber auch zu chronischen Belastungen mit erheblichen Gesundheitseffekten führen, die bis hin zu vorzeitigem Versterben auf Grund von z.B. kardiopulmonalen Erkrankungen oder Lungenkrebs reichen können. Die Holzfeuer-Partikel enthalten unge- wöhnlich viele polyzyklische aromatische Kohlenwasserstoffe (PAK), die krebserregend sind.

Eine starke Belastung mit solchen Teilchen gilt als Risikofaktor für verschiedene akute und chronische Atemwegserkrankungen, Herz-Kreislauf-Erkrankungen und einige Krebserkrankungen.

Kenntnislücken zu gesundheitlichen Aspekten: Es ist bekannt, dass offene Kamine zu einer Belastung der Innenraumluft mit Feinstaub führen können. Bei geschlossenen Kaminen und Kachelöfen sieht die Situation für die Innenraumluft besser aus. Dies setzt aber eine korrekt ausgeführte und dichte Abgasführung sowie das Verwenden abgelagerten, trockenen Holzes voraus. Über die Feinstaub-Belastung sowohl durch offene Kamine als auch durch geschlossene Anlagen im Innenraum (z. B. im Wohnzimmer) liegen bisher nur wenige Erkenntnisse vor [18].

\section{Photovoltaik}

Photovoltaik (PV) bezeichnet die direkte Umwandlung von Sonnenstrahlung in elektrischen Strom. Strahlungsenergie der Sonne wird durch Solarzellen aufgefangen und in Elektrizität umgewandelt.

Die Energiewandlung geschieht mit Hilfe von Solarzellen, die zu sog. Solarmodulen verbunden und in Photovoltaikanlagen eingesetzt werden. Während des Betriebs entstehen keine Nebenprodukte oder Abgase.

Kenntnisstand $z u$ direkten gesundheitlichen Auswirkungen: Direkte gesundheitliche Auswirkungen sind durch das Betreiben der Anlagen als solches nicht $\mathrm{zu}$ erwarten. Allerdings ist der Herstellungsund Recycling- oder Entsorgungsprozess von PV-Anlagen mit zum Teil problematischen Rohstoffen zu nennen.

Mit der raschen Verbreitung der PVAnlagen ist eine Vielzahl unterschiedlicher Solarmodule mit unterschiedlichsten Materialien auf den Markt gelangt. Neben Silicium als Trägersubstanz der Solarzelle wird verstärkt die Produktion von Solarzellen mit den preisgünstigeren Cadmium-Tellurid-Solarzellen (CdTeSolarzellen) betrieben, die aktuell bereits einen Anteil von ca. $10 \%$ ausmachen. Das Material ist aufgrund seiner Eigenschaften sehr gut für die Umwandlung von 
Sonnenlicht in Elektrizität geeignet [19]. Ein Review dreier großer Studien, organisiert vom European Commission's Joint Reseach Center (JRC) bilanziert, dass das Umwelt- und Gesundheitsrisiko von CdTe-Solarzellen bei bestimmungsgemäßer Verwendung minimal ist. Auch die Emissionsprodukte, die während der Lebenszeit der Solarmodule entstehen, sind extrem niedrig [20]. Fthenakis [21] resümiert, dass CdTe-Module kein Gesundheits- und Umweltrisiko während des Gebrauchs und nach dem Recyclingprozess darstellen [21].

Allerdings kann z. B. bei zerbrochenen, der Witterung ausgesetzten Solarmodulen ebenso im Recycling- oder Entsorgungsprozess Cadmium in die Umwelt freigesetzt werden, wodurch die Gesundheit geschädigt werden kann, denn Cadmium ist nachgewiesenermaßen ein humankanzerogener Stoff. Dennoch haben erneuerbare Energien dieser Art, insgesamt betrachtet, eine deutlich bessere Öko-Bilanz gegenüber den fossilen Brennstoffen. Zu diesem Ergebnis kommt eine Lebenszyklus-Analyse internationaler Wissenschaftler und Wissenschaftlerinnen [22].

Kenntnislücken zu gesundheitlichen Aspekten: Die Lebensdauer von Solarzellen ist begrenzt, da sie mit zunehmendem Alter weniger Strom liefern, sie wird für kristalline Solarzellen in Fachkreisen auf durchschnittlich 20-25 Jahre geschätzt. Um die problematischen Stoffe zu recyclen, ist die Einführung eines Rücknahmeprogramms zur Wiederverwendung dringend zu empfehlen $[23,24]$. Bislang gibt es in Deutschland noch keine rechtliche Regelung zur Entsorgung alter Photovoltaikanlagen.

Aspekte zu den indirekten Auswirkungen: Im Gegensatz zu Anlagen, die auf oder in Dächern montiert werden, ist der Landverbrauch bei auf dem Boden installierten PV-Anlagen ein zentraler Kritikpunkt [22]. Hinzu kommt, dass die Problematik der Spiegelung und Blendung von PV-Anlagen auf Dächern durch die zur besseren Energienutzung aufgebrachte Antireflexschicht zwar zum Teil gemindert wird, jedoch zu bestimmten Jahresund Tageszeiten trotzdem als störend und belästigend wirken können [25]. Insgesamt können weitere indirekte gesund- heitsrelevante Aspekte benannt werden (Bodenverdichtung, Bodenversiegelung, Bodenumlagerung und -durchmischung auf Zufahrtsstraßen und Plätzen; Die Einzäunung führt zum Flächenentzug, zur Zerschneidung von Grünräumen; Geräusche, Erschütterungen und stoffliche Emissionen werden z. B. durch Baustellen- und Wartungsverkehr verursacht; Die Überdeckung von Boden durch Solar-Modulflächen führt zur Beschattung, Veränderung des Bodenwasserhaushaltes sowie Bodenerosion. Durch das Aufheizen der Solar-Module wird Wärme an die Umgebung abgegeben; Es können sich elektrische und elektromagnetische Felder bilden).

\section{Wasserkraft}

Wasserkraft ist eine der ältesten Energiequellen, bei der Wasser aufgestaut wird, um Mahl- oder Sägewerke anzutreiben oder Strom mithilfe von Turbinen zu erzeugen. Die Stromerzeugung hat einen hohen Wirkungsgrad wobei weder Schadstoffe noch Abwärme entstehen, die an die Umwelt abgegeben werden.

Kenntnisstand zu direkten gesundheitlichen Auswirkungen: Direkte gesundheitliche Auswirkungen auf den Menschen sind beim Betrieb nicht zu erwarten. Insgesamt schneidet die Wasserkraft von allen anderen fossilen, nuklearen oder neuen Energieträgern aus gesundheitlicher Sicht bei der Betrachtung der Auswirkungen, wie Klimawandel, menschliche Gesundheit, Ozon, Humantoxizität, photochemische Oxidation, Feinstaubentstehung sowie ionisierende Strahlung am besten ab [26].

Kenntnislücken zu gesundheitlichen Aspekten: Keine bekannt

Aspekte zu den indirekten Auswirkungen: Negativ zu beurteilen ist, dass beim Bau eines Wasserkraftwerks/Stausees massiv in die Landschaftsökologie eingegriffen wird. Die negativen Aspekte betreffen vor allem die Landschaftszerstörung, die Flussregulierungen, die Störung der Kontinuität von Fließgewässern, größere Gefahren durch Erdbeben bei großen Stauseen, rasch wechselnde Flusswasserführung, Verminderung der Fischbestände, Umsiedlung von Anwohnerinnen und Anwohnern [27].

\section{Hochspannungsleitungen}

Informationen des Bundesamtes für Strahlenschutz (BfS), der Strahlenschutzkommission (SSK), ein veröffentlichter Artikel [28], sowie ein Teil-veröffentlichtes Papier von [29] bilden die Grundlage der hier wiedergegebenen Hinweise zu den gesundheitlichen Auswirkungen elektromagnetischer Felder.

Zur Übertragung elektrischer Energie über große Entfernungen werden Hochund Höchstspannungsleitungen im Verbundnetz eingesetzt. Dabei werden besonders hohe elektrische Spannungen angelegt (min. $60 \mathrm{kV}$ (Kilovolt) bis ca. $1000 \mathrm{kV}$ ). So kann ohne allzu hohe Leistungsverluste mit moderater Stromstärke eine hohe elektrische Leistung übertragen werden. Die Spannung wird dabei umso höher gewählt, je höher die übertragene Leistung und je länger die Leitung ist. Im Umfeld entsteht dabei ein niederfrequentes elektrisches und magnetisches Feld. Das elektrische Feld lässt sich leicht durch Bäume, Erdreich oder Hauswände abschirmen und es dringt kaum in den menschlichen Körper ein. Magnetische Felder besitzen die Eigenschaft, Hauswände und auch den menschlichen Körper zu durchdringen und können nicht ohne weiteres abgeschirmt werden.

In Deutschland sind die meisten Hochspannungsleitungen Freileitungen, es gibt wenige im Boden verlegte Hochspannungs-Erdkabel. Im Vergleich zu Freileitungen verursachen Erdkabel wesentlich höhere Kosten, erfordern aufwändige Tiefbauarbeiten in einer den Freileitungen vergleichbaren Trassenbreite und die Freihaltung des Trassenbereiches für etwaige Reparaturarbeiten aufgrund von Schäden, die durch Korrosion, Überspannungen, Übererwärmungen oder durch äußere mechanische Einwirkungen verursacht werden können. Die elektrischen Verlustleistungen verursachen Bodenerwärmungen, die im Trassenbereich zur Bodenaustrocknung und zur Beeinträchtigung des Biotops führen können [30].

Mit dem Umstieg auf erneuerbare Energien ergibt sich die Notwendigkeit, große Strommengen in Deutschland von Nord nach Süd zu transportieren. Die örtliche Festlegung der sich daraus ergebenden Neuerrichtung und Verstärkung von 
Hochspannungsleitungen ist noch nicht erfolgt.

Kenntnisstand $z u$ direkten gesundheitlichen Auswirkungen: Bislang wurden überwiegend Hochspannungsleitungen mit Wechselstromübertragung (HWÜ) betrieben, deren Freileitungen ein niederfrequentes elektrisches bzw. magnetisches Feld erzeugen.

Auf den Web-Seiten des Bundesamt für Strahlenschutz werden die Gesundheitliche Aspekte niederfrequenter elektrischer Feldintensitäten bei Wechselstrom dargestellt (Quelle: Bundesamt für Strahlenschutz http://www.bfs.de/SharedDocs/ Bilder/BfS/DE/emf/nff/wirkungen-felder. jpg?_blob=poster\&v=2 (Abruf November 2016).

Ferner werden definierte Wirkungen (z.B. Einflüsse auf das Krebsgeschehen und auf neurodegenerative Erkrankungen) wissenschaftlich diskutiert:

- Es besteht ein epidemiologischer Zusammenhang zwischen der Exposition gegenüber niederfrequenten Magnetfeldern (NF MF) und einer Häufung kindlicher Leukämien [31, 32].

- Die IARC (International Agency for Research on Cancer) stufte 2002 niederfrequente Magnetfelder in die Gruppe 2b (möglicherweise krebserregend) ein [46].

- Wissenschaftliche Ergebnisse sind heterogen und zeigen eine geringe Evidenz bezüglich des Zusammenhangs von NF MF und Hirntumoren, Leukämien bei Erwachsenen sowie amyotropher Lateralsklerose [33] und Fehlgeburten. Solche Zusammenhänge werden überwiegend für unwahrscheinlich gehalten.

- Übereinstimmend wird der Zusammenhang von NF EMF mit dem Auftreten von Brustkrebs, Alzheimer- Erkrankungen und dem Auftreten von Depressionen abgelehnt.

- Die Existenz einer Elektrosensibilität ist aktuell nicht wissenschaftlich belegt [28].

Die Strahlenschutzkommission empfiehlt, dass an Orten, die Trägerinnen und Trägern von elektronischen Implantaten (wie Herzschrittmachern oder implantierten Defibrillatoren) zugänglich sind und an denen ein Ausweichen nicht zumutbar ist, Werte für das niederfrequente Magnetfeld von 10 bzw. $15 \mu$ T nicht überschritten werden sollten [34].

Neben den bekannten Hochspannungsleitungen mit Wechselstromübertragung (HWÜ) sollen auch die für lange Distanzen günstigeren Hochspannungsleitungen mit Gleichstromübertragung (HGÜ) eingesetzt werden. Hochspannungsleitungen mit Gleichstromübertragung stellen eine neuere Technik dar. Hier liegt ein statisches elektrisches und magnetisches Gleichfeld vor.

Derzeit wird davon ausgegangen, dass die statischen Magnetfelder von HGÜLeitungen in unmittelbarer Trassennähe in etwa die Größenordnung des natürlichen Erdmagnetfeldes erreichen werden. Dieses hat in Deutschland eine magnetische Flussdichte von etwa 50 Mikrotesla. Über die elektrischen Feldstärken von HGÜ-Freileitungen liegen noch wenige Informationen vor; für sie gilt allerdings - da statische elektrische Felder nicht in den Körper eindringen - auch keine Grenzwertbeschränkung [35]. Im Unterschied zu den Hochspannungs-Wechselstrom-Leitungen können sich um die Leiter größere Raumladungswolken geladener Teilchen ausbilden, die zur Ionisation der Luft führen und chemische Verbindungen wie Ozon und Stickoxide entstehen lassen können. Bei HGÜ treten im Vergleich zu Hochspannungs-Wechselstrom-Leitungen deutlich höhere elektrische Bodenfeldstärken auf und die elektrischen Gleichfelder können sich durch Windverfrachtungen der Ladungswolke über größere Bereiche erstrecken. Sowohl die elektrische Feldstärke als auch die magnetische Flussdichte nehmen mit dem Abstand von der Quelle erheblich ab.

Im Erdboden verlegte HGÜ Kabelleitungen erzeugen an der Erdoberfläche praktisch nur magnetische Gleichfelder. Weder äußere elektrische Felder noch Koronaentladungen spielen hier eine Rolle (und damit auch kein Ozon und keine Stickoxide).

Die 26. Bundesimmissionsschutzverordnung sieht für Magnetfelder, die durch HGÜ verursacht werden, einen Grenzwert von 500 Mikrotesla vor. Die zu erwartenden Flussdichten der HGÜ liegen selbst in Trassenmitte mit 45 Mik- rotesla weit unterhalb der (internationalen) Grenzwertempfehlung. Sie ähneln der Flussdichte des Erdmagnetfeldes, das in Mitteleuropa etwa 50 Mikrotesla beträgt.

Für Gleichstromübertragung sind biologische Effekte und damit unmittelbare gesundheitliche Wirkungen statischer Felder nur bei sehr hohen Magnetfeldstärken bekannt. Bei den niedrigen Magnetfeldstärken in der Umgebung von HGÜ-Leitungen oder Konvertern sind daher keine gesundheitlich negativen Wirkungen zu erwarten. Schwächere Magnetfelder können aber ein mittelbares Risiko darstellen, weil sie Kräfte auf magnetisierbare Objekte ausüben und Implantate beeinflussen können [35].

Kenntnislücken zu gesundheitlichen Aspekten: Forschungsbedarf besteht insbesondere zur Frage, ob von magnetischen Gleichfeldern der HGÜ ein zusätzliches Risiko für die Krebsentstehung ausgeht.

Aspekte zu den indirekten Auswirkungen: Die Hochspannungsleitungen führen zu Beeinträchtigung der landschaftlichen Integrität, zerschneiden mit ihren Trassen die Landschaft und haben auch Auswirkungen auf die Tierwelt (z.B. Vögel) und den Tourismus.

\section{Energiespeicher}

Zur Gewährleistung der Versorgung bei wind- oder sonnenarmem Wetter wird langfristig ein Mix verschiedener Speichertechnologien erforderlich sein. Die Bundesregierung hat im Rahmen des 6 . Energieforschungsprogramms 200 Mio. Euro für die „Förderinitiative Energiespeicher" bereitgestellt. Dabei sollten gesundheitliche Aspekte bereits in der Entwicklung bedacht werden.

Kenntnisstand $z u$ direkten gesundheitlichen Auswirkungen: Die direkten Auswirkungen werden zum einen durch die $\mathrm{Zu}$ - und Ableitungen der Hochspannungsleitungen ausgelöst (siehe dort), sowie durch die vorhandenen elektrischen und magnetischen Felder am Energiespeicher selbst.

Kenntnislücken zu gesundheitlichen Aspekten: Forschungsbedarf besteht zu den biologischen Wirkungen der elektrischen 
und magnetischen Felder in Bezug auf die Entstehung von Krebs.

\section{Energetische Sanierung/ Wärmedämmung}

Energiesparen ist ein wichtiges Anliegen der Umweltpolitik, denn es vermindert u. a. den Ausstoß klimaschädlicher Gase. Energiesparende Maßnahmen im Gebäudebereich führen häufig dazu, dass bei Neubauten und bei der Sanierung von Altbauten die Gebäudehülle „luftdichter“ wird. Der verringerte Luftaustausch zwischen dem Innenraum und der Außenluft reduziert Wärmeverluste und spart daher auch Energiekosten.

Kenntnisstand zu direkten gesundheitlichen Auswirkungen: Als erstes sind gesundheitliche Auswirkungen des Dämmmaterials selbst $\mathrm{zu}$ betrachten, beim Herstellungsprozess, bei der Verarbeitung und bei der Entsorgung. Mineralwolle oder Holzfasern sowie Wärmedämmverbundsysteme als auch gefüllte Ziegelsteine werden bei der Dämmung von Einfamilienhäusern eingesetzt, überwiegend wird jedoch das preisgünstigere aber äußerst problematische Polystyrol verwendet. Die Marktdurchdringung von Dämmstoffen aus nachwachsenden Rohstoffen ist noch gering, während Dämmstoffe aus Styropor (Polystyrol) einen Marktanteil von ca. 50\% halten und jene aus Mineralwolle ca. $39 \%$, wie aus dem privaten „Branchenradar Dämmstoffe 2014“ [44] hervorgeht. Polystyrol muss vor der Verwendung als Dämmplatte vielfach mit verschiedenen Flammschutzmitteln behandelt werden, um eine schwerere Entflammbarkeit $\mathrm{zu}$ erreichen und bei der Bearbeitung mit Heißdraht können giftige Dämpfe entstehen. Die diesbezüglichen Grenzwerte (Innenraumrichtwert I und II) für Wohnbereiche der angrenzenden Anwohner sind beispielsweise für Styrol festgelegt, wobei der Innenraumrichtwert II nicht überschritten werden sollte (UBA Ausschuss für Innenraumrichtwerte [48]). Richtwerte sind: RW I: $0.03 \mathrm{mg} / \mathrm{m}^{3}$ und RW II $0.3 \mathrm{mg} / \mathrm{m}^{3}$ [36].

Im Brandfall können giftiges Kohlenmonoxid sowie zum Teil andere giftige Stoffe freigesetzt werden.
Die isolierten Fassaden werden auch häufig mit Chemikalien behandelt, um Algenbewuchs zu verhindern. Studien zufolge geraten diese Stoffe nach und nach mit dem Regen ins Grundwasser und das angrenzende Erdreich [37].

Problematisch ist bislang die zurzeit noch nicht geklärte Entsorgung in Müllverbrennungsanlagen. Expertinnen und Experten des Umweltbundesamtes fordern eine gesetzliche Lösung für den sogenannten selektiven Rückbau, bei dem die Materialien einzeln entsorgt werden können.

Des Weiteren werden Gebäude durch die Dämmung luftdichter und als Folge dieses verminderten Luftaustausches kann es, bei mangelhafter Lüftung, zu Beeinträchtigungen der Innenraumluftqualität kommen. Emissionen aus Bauprodukten und Materialien der Innenraumausstattung sowie Feuchtigkeit, die beispielsweise beim Duschen und Kochen entsteht, muss durch geeignete Lüftungskonzepte aus dem Hausinneren befördert werden. Erhöhte Konzentrationen flüchtiger organischer Verbindungen und Schimmelpilzwachstum auf feuchten Wänden können - in Abhängigkeit vom jeweiligen Substrat - die Folge sein und dadurch negative gesundheitliche Folgen mit sich bringen.

Auch wenn die kausale Wirkungskette zwischen einem konkreten Schimmelpilzbefall mit einem Gemisch aus verschiedenen Schimmelpilzarten und mit ihm einhergehenden Milben- und bakteriellen Belastungen noch nicht gänzlich entschlüsselt ist, bleibt dennoch unbestritten, dass Schimmelpilzbefall Infektionen, Sensibilisierungen und Allergien, toxische Wirkungen sowie Geruchswirkungen und Befindlichkeitsstörungen auslösen kann. Diese sind abhängig von der gesundheitlichen Anfälligkeit der Menschen im Innenraum für Schimmelpilzexpositionen.

Nützliche Hinweise können dem Schimmelleitfaden des Umweltbundesamtes und den Leitlinien des AWMF entnommen werden $[37,38]$ :

Kenntnislücken $z u$ gesundheitlichen Aspekten: Nicht systematisch untersucht wurde die Dämmung der verschiedensten Baukörper auf ihre Kompatibilität mit den sie umhüllenden Dämmmaterialien (z. B. lassen sich Lehmhäuser nicht mit Polysty- rol dämmen, da die Feuchtigkeit der Baukörper sich nicht allein durch Lüften aus dem Bau heraustransportieren lässt und zusätzlich immer wieder neue Feuchtigkeit durch die Lehmwände hochgesogen wird).

Festgelegte Innenraumrichtwerte beruhen auf definierten Luftwechselraten, die durch die energetische Sanierung drastisch abnehmen. Das kann zu einer Akkumulation von Schadstoffen aus den in der Wohnung vorhandenen Materialien führen. Vielfach ist eine regelmäßige Lüftung (mehrmals täglich je 5-10 Minuten querlüften) für das Aufrechterhalten eines gesunden Wohnklimas nicht möglich (Abwesenheit tagsüber, Schlaf nachts, in der kalten Jahreszeit auskühlen der Wohnung, da kein Wärmeaustausch stattfindet). In Neubauten und Null-Emissionshäusern mit einer integrierten Lüftungsanlage führt die Dämmung zu weniger negativen Auswirkungen auf die Bewohnerinnen und Bewohner.

Insgesamt fehlen eine vorherige $\mathrm{Ab}$ klärung der oben geschilderten Auswirkungen und eine lüftungstechnische Unterweisung, wie die Auswirkungen der Dämmung durch Lüftung verhindert werden können. $\mathrm{Ob}$ eine Dämmung für spezielle Baukörper überhaupt sinnvoll erscheint, lässt sich nur beantworten, wenn nicht nur nach energetischen sondern auch nach gesundheitlichen Aspekten in diesem mehrdimensionalen Entscheidungsprozess abgewogen wird. Es sollte jedoch darüber diskutiert werden, ob die aus energetischen Gründen wünschenswerte Luftdichtheit aus hygienischer Sicht ebenso günstig ist.

Nach der Dämmung und der erhöhten Dichtigkeit des Gebäudes bleibt zu beobachten, wie sich die Materialien im Hinblick auf Feuchtregulation bzw. Aufstieg der Feuchte (beispielsweise bei älteren Gebäuden ohne bauliche Maßnahmen zum Schutz vor aufsteigender Feuchte) verhalten. Der geringere Luftaustausch hat auch Auswirkungen auf das Innenraumklima, z.B. durch die Abgabe von Schadstoffen aus den (alten) Baustoffen und deren mögliche Akkumulation in der Innenluft. Sofern ein Luftaustausch bei abgedichteten Gebäuden durch Lüftungsanlagen geregelt wird, fehlen diesbezügliche Erkenntnisse zur Nutzung im privaten Sektor, der 
im Hinblick auf Wartung und Filteraustausch der Verantwortung des Eigentümers unterliegt und weniger reguliert ist wie in öffentlichen Gebäuden.

Daneben bedarf es noch großer Anstrengungen, die Entsorgung der Dämmmaterialien nicht nur gesetzlich zu regeln, sondern auch geeignete Entsorgungstechniken zu entwickeln, um alle bei der Verbrennung entstehenden und vorhandenen Schadstoffe herausfiltern zu können.

Aspekte zu den indirekten Auswirkungen: Bauliche Veränderungen von historischen Fassaden zu uniformen Putzfassaden, Verschwinden von Klinker- und Fachwerkbauten. Bei denkmalgeschützten Fassaden kann eine Innendämmung vorgenommen werden, die ihrerseits nicht unproblematisch ist: Innendämmung geschieht auf Kosten der Wohnfläche, und es besteht die Gefahr der Schimmelpilzbildung durch eine Taupunktverschiebung. Zudem kann das zum Teil massive Mauerwerk nicht mehr als Wärmespeicher fungieren, da es sich außerhalb des gedämmten Raumes befindet. Es besteht zusätzlich eine Korrosions- und Frostgefahr der in der Wand verlegten Wasserund Heizungsrohre.

\section{Betrachtung gesundheitlicher Aspekte der im Rahmen der Energiewende diskutierten Energieträger/Energieeinspa- rungsmaßnahmen}

Bislang sind die mit der Energiewende verbundenen Chancen und Risiken für die menschliche Gesundheit der Bevölkerung noch nicht ausreichend sichtbar aufgegriffen und überprüft worden. Dies wäre notwendig, um eventuelle Umweltoder Gesundheitsschäden frühzeitig identifizieren zu können und falls erforderlich Gegenmaßnahmen einzuleiten.

Eine Gesamt-Bilanzierung solcher Betrachtungen könnte mit Hilfe der Gesundheitsfolgenabschätzung bewerkstelligt werden, einem Methodeninstrument zur verstärkten Berücksichtigung der gesundheitlichen Auswirkungen von politischen Maßnahmen in verschiedensten Sektoren. Dieser Prozess geht strukturiert der Frage nach, welche weitreichenden gesundheitlichen Konsequenzen ein solches (politisches) Vorhaben voraussichtlich nach sich ziehen wird. Da eine solche Analyse der Gesundheitsauswirkungen zur Verbesserung der Bevölkerungsgesundheit beitragen kann, ist sie ein wichtiger Pfeiler im Rahmen einer "Health in all Policies“Strategie.

\section{Das Instrument "Gesundheitsfol- genabschätzung"}

Bislang sind in Deutschland etablierte Instrumente einer Gesundheitsfolgenabschätzung (GFA) (engl. Health Impact Assessment (HIA)) weitgehend ungenutzt geblieben. „Die Gesundheitsfolgenabschätzung als ein systematischer Prozess zur Analyse und Bewertung geplanter politischer Vorhaben hinsichtlich der möglichen positiven sowie negativen Auswirkungen auf die Gesundheit der Bevölkerung und die Verteilung der gesundheitlichen Auswirkungen innerhalb der Bevölkerung", könnte folglich auch in der Energie- und Ressourcenpolitik wesentlich dazu beitragen, die gesundheitlichen Auswirkungen der verschiedenen Technologien und Maßnahmen qualitativ und quantitativ zu bestimmen.

Aus umweltmedizinischer Sicht sollte in erster Linie sowohl die Definition und Benennung der umweltmedizinisch relevanten Aspekte bzw. der diesbezüglichen Kenntnislücken und die Notwendigkeit, diese zu schließen, das Ziel sein. Erst in einem weiteren Schritt sollte die Gesundheitsfolgenabschätzung als Instrument gestärkt werden. Werden bei vorhandener Datenlage Kenntnisse zu gesundheitlichen Auswirkungen berücksichtigt, ist dieses Instrument auch für umweltmedizinische Belange aussagekräftig.

Zusätzlich könnte mittels konsequentem Einsatz einer GFA das Verständnis für den Stellenwert, den Gesundheit innerhalb der Energiepolitik und für die $\mathrm{Zu}$ sammenarbeit der unterschiedlichen Entscheidungsträger hat, erheblich gestärkt und Grundlagen möglicher Präventionsstrategien geschaffen werden.

Das Instrument Gesundheitsfolgenabschätzung hat sich in der Vergangenheit bereits vielfach als aussichtsreiche Möglichkeit zur konkreten Umsetzung der gesundheitspolitischen Strategie „Gesundheit in allen Politikfeldern“ bewährt bzw. ist mit dieser Strategie eng verbunden [40]. Dabei kann der partizipatorische Prozess auch als ein strategisches Health Impact Assessment auf der Bundesebene genutzt werden.

Im Rahmen einer GFA werden konkrete (politische) Vorhaben systematisch auf ihre Auswirkungen auf die Gesundheit der Bevölkerung untersucht. Auf EU-Ebene wird die GFA als zukunftsträchtiges Instrument zur verstärkten Berücksichtigung von Gesundheitsaspekten in unterschiedlichen Politikzusammenhängen gesehen und die Anwendung sowohl auf Ebene der EU-Institutionen als auch in den Mitgliedstaaten empfohlen [41]. Das Regionalbüro der Weltgesundheitsorganisation (WHO) in Europa hat die Etablierung von GFA in den Mitgliedsländern als eines der Gesundheitsziele 2020 definiert [42] und einen entsprechenden Schwerpunkt im Netzwerk „Gesunde Städte“ verankert. Dabei können sowohl strategische Ausrichtungen der Methode auf Bundesebene als auch projektbezogene Gesundheitsfolgenabschätzungen auf regionaler Ebene durchgeführt werden.

Der GFA (oder HIA)-Ansatz gibt die Abfolge der Verfahrensschritte vor und bedient sich mehrerer Methoden wie Literaturrecherche, Expertenanhörungen, Stakeholderbeteiligung sowie mathematische Modellierung. Im Vorfeld werden die Aufgaben in einer Steuerungs- oder Lenkungsgruppe festgelegt und der Kreis der Beteiligten sowie die Beschreibung der zu bearbeitenden Wirkungsbereiche ausgewählt. Die Methode fügt damit eine Vielzahl von Informationen aus diversen Quellen zu einer stimmigen evidenzbasierten Bewertung und Prognose von gesundheitsrelevanten Fragen bzw. potenziellen Gesundheitsbeeinträchtigungen zusammen und bildet damit die Grundlage von Entscheidungen. Zu den Verfahrensschritten zählen Screening, Scoping, die eigentliche Analyse/Bewertung (Appraisal) sowie ein Bericht inkl. Empfehlungen, Evaluation und Monitoring.

Insbesondere bei innovativen neu einzuführenden Vorhaben (z. B. Verwendung neuer Materialien, neue Energieträger ohne Erfahrungswerte) fehlen naturgemäß aussagekräftige wissenschaftliche Untersuchungen $\mathrm{zu}$ den gesundheitli- 
chen Effekten. Hier können im Rahmen von Defizitanalysen Wissens- und Forschungsbedarfe aufgezeigt werden. Da das Ziel einer GFA eine umfassende Analyse möglicher Gesundheitsfolgen darstellt, können auch die positiven gesundheitlichen Aspekte, die durch einen langfristigen Ausstieg aus der Kern- oder Kohleenergie entstehen, in die Bilanzierung einbezogen werden.

Dessen ungeachtet fehlen bislang langfristig angelegte epidemiologische Studien, die im Kohortenansatz mögliche $\mathrm{Zu}$ sammenhänge beleuchten könnten. Eine evidenzbasierte epidemiologische Bestätigung eines Kausalzusammenhangs ist oft, je nach Risikofaktor und Gesundheitsendpunkt, erst nach einer langen Latenzzeit zu erwarten. Nach der Einführung neuer Maßnahmen könnten begleitende Studien deshalb zu frühzeitigen Entscheidungen und zur Verhinderung von Gesundheitsschäden beitragen.

Eine GFA bietet sich grundsätzlich für ein großes Spektrum von Möglichkeiten an. Die Energiewende als Gesamtprozess mit einer GFA zu beurteilen ist jedoch nur in einem interdisziplinären Ansatz unter Berücksichtigung umweltmedizinischer, technischer, ingenieurswissenschaftlicher und anderer Expertise möglich und erfordert den Einsatz von finanziellen/personellen Ressourcen. Deshalb könnte es sich im Rahmen der Energiewende anbieten, die jeweiligen Maßnahmen zunächst getrennt voneinander $\mathrm{zu}$ betrachten und hierbei nach heutigem wissenschaftlichem Stand relevante Aspekte prioritär zu behandeln. Um vor einer möglichen wissenschaftlichen interdisziplinären Evaluation frühzeitig gesundheitsbezogene Aspekte in den Prozess der Energiewende zu inkludieren, empfiehlt es sich aus Sicht der Kommission, umweltmedizinische Expertise in die technischen Gremien zur Normengebung einzuholen. Auch sollten die Maßnahmen der Energiewende bzgl. deren Effektivität begleitend beobachtet werden, um die Bewertung nicht in erster Linie auf Hochrechnungen zu stützen. Im Hinblick auf die politisch intendierte Förderung von Maßnahmen zur Energiewende sollten Maßnahmen zur Produktförderung mit gesundheitlichen Aspekten verknüpft werden; dies kann als innovativer Aspekt zur nachhaltigen Stärkung des Wirtschaftsstandorts Deutschland beitragen.

Die Stellungnahme wurde von der Kommission Umweltmedizin am Robert Koch-Institut per Umlaufbeschluss im April 2016 verabschiedet.

\section{Federführung}

Priv.-Doz. Dr. med. A. Heutelbeck (Universitätsmedizin Göttingen (UMG), Institut für Arbeits-, Sozial- und Umweltmedizin), Prof. Dr. C. Hornberg (Universität Bielefeld, Fakultät für Gesundheitswissenschaften), Prof. Dr. C. Herr (Bayerisches Landesamt für Gesundheit und Lebensmittelsicherheit), Prof. Dr. W. Dott (Universitätsklinikum Aachen, Institut für Hygiene und Umweltmedizin), Prof. Dr. Th. Eikmann (Universität Gießen, Institut $f$. Hygiene u. Umweltmedizin), Dr. M. Schümann (vormals Behörde für Gesundheit und Verbraucherschutz Freie und Hansestadt Hamburg)

\section{Kommissionsmitglieder}

Prof. Dr. K.-C. Bergmann (Allergie-Centrum, Charité Universitätsmedizin Berlin), Dr. J. Heinrich (Helmholtz Zentrum München, Epidemiologie I), Prof. Dr. B. Hoffmann (Heinrich-Heine-Universität Düsseldorf, Institut für Arbeits-, Sozial- und Umweltmedizin), Prof. Dr. V. Mersch-Sundermann (Universität Freiburg, Institut Krankehaushygiene u. Umweltmedizin), Prof. Dr. D. Nowak (LMU München, Klinikum Innenstadt, Institut u. Poliklinik für Arbeits- und Umweltmedizin), Dr. M. Otto (Gemeinnützige Kinderumwelt $\mathrm{GmbH}$ ), Prof. Dr. A. Peters (Helmholtz Zentrum München, Epidemiologie II), Prof. Dr. G. A. Wiesmüller (Gesundheitsamt Köln)

\section{Ständige Gäste}

Dr. U. Winkler, K. Höppner M. Sc. (Bundesministerium für Gesundheit), J. Küllmer (Bundesministerium für Umwelt, Naturschutz, Bau und Reaktorsicherheit), Dr. A. Gies, Dr. W. Straff, Dr. A. Neumann, Dr D. Plaß (Umweltbundesamt), Dr. H. Desel (Bundesinstitut für Risikobewertung, Berlin), Dr. Th. Jung (Bundesamt für Strahlenschutz), Dr. Th. Ziese, Dr. M. Fa- ber, Prof. Dr. K. Stark (Robert Koch-Institut), Dr. H. Niemann (Geschäftsstelle der Kommission Umweltmedizin am Robert Koch-Institut), Dr. F. Benkwitz (Vorsitzender der Länderarbeitsgruppe umweltbezogener Gesundheitsschutz, LAUG)

\section{Kontakt über die Geschäftsstelle am RKI}

Robert Koch-Institut - Geschäftsstelle der Kommission Umweltmedizin

Fachgebiet 24: Gesundheitsberichterstattung

Postfach 6502 61; D-13302 Berlin

E-Mail: Umweltmedizinkommission(at) rki.de

\section{Literatur}

1. BMWI (2010) Energiekonzept für eine umweltschonende, zuverlässige und bezahlbare Energieversorgung. Bundesministerium für Wirtschaft und Technologie (BMWi). Bundesministerium für Umwelt, Naturschutz und Reaktorsicherheit (BMU), Berlin

2. Raaschou-Nielsen O, Andersen ZJ, Beelen R, Samoli E, Stafoggia M, Weinmayr G, Hoffmann B, Fischer P, Nieuwenhuijsen MJ, Brunekreef B, Xun WW, Katsouyanni K, Dimakopoulou K, Sommar J, Forsberg B, Modig L, Oudin A, Oftedal B, Schwarze PE, Nafstad P, De Faire U, Pedersen NL, Östenson CG, Fratiglioni L, Penell J, Korek M, Pershagen G, Eriksen KT, Sørensen M, Tjønneland A, Ellermann T, Eeftens M, Peeters PH, Meliefste K, Wang M, Bueno-de-Mesquita B, Key TJ, de Hoogh K, Concin H, Nagel G, Vilier A, Grioni S, Krogh V, Tsai M-Y, Ricceri F, Sacerdote C, Galassi C, Migliore E, Ranzi A, Cesaroni G, Badaloni C, Forastiere F, Tamayo I, Amiano P, Dorronsoro M, Trichopoulou A, Bamia C, Vineis P, Hoek G (2013) Air pollution and lung cancer incidence in 17 European cohorts: prospective analyses from the European Study of Cohorts for Air Pollution Effects (ESCAPE). Lancet Oncol 14(9):813-822

3. HEAL (2013) The unpayed health bill - how coal power plants make us sick. A report from the Health and Environment Alliance. http://www. env-health.org/IMG/pdf/heal_report_the_unpaid_health_bill_how_coal_power_plants_ make_us_sick_final.pdf. Zugegriffen: 15. Febr. 2016

4. Preiss P, Roos J, Friedrich R (2013) Assessment of Health Impacts of Coal Fired Power Stations in Germany by Applying EcoSenseWeb. https://www. greenpeace.de/sites/www.greenpeace.de/files/ publications/greenpeace-studie-tod-aus-demschlot-s01652.pdf. Zugegriffen: 15. Febr. 2016

5. BMWI/AGEE-Stat (2015) http://www.erneuerbareenergien.de/EE/Redaktion/DE/Downloads/ zeitreihen-zur-entwicklung-der-erneuerbarenenergien-in-deutschland-1990-2014-excel.xlsx;j sessionid=5D10747A07CED856AB55A4606175 67F8?_blob=publicationFile\&v=2. Zugegriffen: Nov. 2016 
6. Hübner G, Pohl J (2010) Akzeptanz und Umweltverträglichkeit der Hinderniskennzeichnung von Windenergieanlagen. Abschlussbericht zum BMUForschungsvorhaben (FKZ: 03MAP134).

7. Møller H, Pedersen CJ (2010) Lavfrekvent støj fra store vindmøller. http://vbn.aau.dk/ files/52554719/Moeller_og_Pedersen_Lavfrekvent_stoej_fra_store_vindmoeller.pdf. Zugegriffen: 15. Febr. 2016

8. Bayerisches Landesamt für Umwelt, Bayerisches Landesamt für Gesundheit und Lebensmittelsicherheit (2014) Windenergieanlagen - beeinträchtigt Infraschall die Gesundheit? http://www. Ifu.bayern.de/umweltwissen/doc/uw_117_windkraftanlagen_infraschall_gesundheit.pdf.Zugegriffen: 15. Febr. 2016

9. LUBW (2014)Landesanstalt für Umwelt, Messungen und Naturschutz Baden-Württemberg - Tieffrequente Geräusche und Infraschall von Windkraftanlagen und anderen Quellen - Zwischenbericht über Ergebnisse des Messprojekts 2013-2014.

10. Krahé $D$, Schreckenberg D, Ebner F, Eulitz $C$, \& Möhler U. (2014). Machbarkeitsstudie zu Wirkungen von Infraschall. Studie im Auftrag des Umweltbundesamtes. UBA Texte, 20, 2014.

11. Schomer PD, Erdreich J, Pamidighantam PK, Boyle $J H$ (2015) A theory to explain some physiological effects of the infrasonic emissions at some wind farm sites. J Acoust Soc Am 137(3):1356-1365

12. Broekel T (2015) Gone with the wind? The impact of wind turbines on tourism demand. Energy Policy 86:506-519. doi:10.1016/j.enpol.2015.08.005

13. Wings TK, Dott W (2015) Ausbreitung von Antibiotika, Mikroorganismen und Resistenzen aus der Intensiv-Tierhaltung und deren Minimierung in Biogasanlagen. http://www.vivis.de/phocadownload/2015_is/2015_IS_297-310_Dott_Wings.pdf. Zugegriffen: 15. Febr. 2016

14. Sigsgaard T, Forsberg B, Annesi-Maesano I, Blomberg A, Bølling A, Boman C, Hirvonen MR (2015) Health impacts of anthropogenic biomass burning in the developed world. Eur Respir J 46:1577-1588. doi:10.1183/13993003.01865-2014

15. UBA-Ausschuss für Innenraumrichtwerte (2014) Gesundheitshygiene: gesundheitlich hygienische Beurteilung von Geruchsstoffen in der Innenraumluft mit Hilfe von Geruchsleitwerten - ein Verfahren zur Ableitung von Geruchsleitwerten. Bundesgesundheitsblatt 2014(57):148-153

16. Reifferscheid F, Krause J, Kühn D, Wirtz S (2013) Biogas-Energiewende mit neuen Risiken für die Notfallmedizin. Notarzt 29:3-7

17. UBA (2015) Luftqualität 2014 - vorläufige Auswertung. http://www.umweltbundesamt.de/ publikationen/luftqualitaet-2014. Zugegriffen: 15. Febr. 2016

18. Behnke A, Straff W (2007) Die Nebenwirkungen der Behaglichkeit: Feinstaub aus Kamin und Holzofen. Umid Umweltmedizinischer Informationsdienst 1:36-39

19. Bundesverband Solarwirtschaft (2009) Cadmium - Tellurid (CdTe) - Photovoltaiktechnologie. http:// www.solarwirtschaft.de/fileadmin/content_files/ Hintergrund_CdTe_0309.pdf. Zugegriffen: 15 . Febr. 2016

20. JRC (2005) Joint Research Center: Summary Report, Peer Review of Major Published Studies on the Environmental of Cadmium-Telluride (CdTe). http://re.jrc.ec.europa.eu/refsys/pdf/Report $\% 20$ Summary-peer\%20review.pdf. Zugegriffen: Nov. 2016

21. Fthenakis V (2002) Could CdTe PV Modules Pollute the environment? https://www.bnl.gov/pv/files/ pdf/art_164.pdf. Zugegriffen: 15. Febr. 2016

22. Hertwich EG, Gibon T, Bouman EA, Aevesen $A$ Suh S, Heath GA, Bergesen JD, Ramirez A, Vega MI, Lei S (2014) Integrated life-cycle assessment of electricity-supply scenarios confirms global environmental benefit of low-carbon technologies. Proc Natl Acad Sci. www.pnas.org/cgi/ doi/10.1073/pnas.1312753111

23. Zimmermann T, Gößling-Reisemann S (2014) Recycling potentials of critical metals - analyzing secondary flows from selected applications. Resources 3(1):291-318

24. Kummer $B$ (2011) RESOLAR-Projekt mit interessanten Ergebnissen abgeschlossen. http://umweltmagazin.de/libary/common/Langfassung.pdf. Zugegriffen: 10. März 2015

25. Rose T, Wollert A (2015) The dark side of photovoltaic - 3D simulation of glare assessing risk and discomfort. Environ Impact Assess Rev 52(2015):24-30

26. Treyer K, Bauer C, Simons A (2014) Human health impacts in the life cycle of future European electricity generation. Energy Policy 74:S31-S44

27. Kumar D, Katoch SS (2014) Sustainability indicators for run of the river (RoR) hydropower projectsin hydro rich regions of India. Renew Sustain Energy Rev 35(2014):101-108

28. Kappos A (2016) Geht vom Ausbau elektrischer Hochspannungsleitungen eine Gefahr für die menschliche Gesundheit aus? Umw Hyg Arbeitsmed 21:89-93

29. Hermann N (2014) Chancen und Risiken der Energiewende aus Gesundheitssicht. CME - Fortbildung zum Expertenworkshop 2014. Das Fortbildungsmodul beruht auf Vorträgen im Rahmen des Expertenworkshops "Chancen und Risiken der Energiewende aus Gesundheitssicht", der am 08. Oktober 2014 in Kooperation zwischen der Bundesärztekammer, der Gesellschaft für Hygiene, Umweltmedizin und Präventivmedizin (GHUP) sowie der Health and Environment Alliance (HEAL) stattfand. Teilweise veröffentlicht in: Hermann N, Eikmann T, Herr C, Huscher J, Kirschbaum B Myck T, Suchenwirth R, Twardella D (2014) Neues Fortbildungsmodul zu "Chancen und Risiken der Energiewende aus Gesundheitssicht". Umw Fre Arbeitsmed 20(4):171-173

30. EFZN (2011) Energieforschungszentrum Niedersachsen: Ökologische Auswirkungen von 380-kVErdleitungen und HGÜ-Erdleitungen. BMU-Studie 2011.

31. Olsen J, Nielsen A, Schulgen G (1993) Residence near high voltage facilities and risk of cancer in children. Brit Med J 307:891-895

32. Pedersen C, Johansen C, Schüz J, Olsen JH, Raaschou-Nielsen O (2015) Residential exposure to extremely low-frequency magnetic fields and risk of childhood leukaemia, CNS tumor and lymphoma in Denmark. Br J Cancer 113:1370-1374

33. Kheifets L, Bowman JD, Checkoway $H$, Feychting M, Harrington JM, Kavet R, Marsh G, Mezei G, Renew DC, van Wijngaarden E (2009) Future needs of occupational epidemiology of extremely low frequency electric and magnetic fields: review and recommendations. Occup Environ Med 66(2):72-80

34. Strahlenschutzkommission (2013) Biologische Effekte der Emissionen von HochspannungsGleichstromübertragungsleitungen (HGÜ), Empfehlungen der Strahlenschutzkommission mit wissenschaftlicher Begründung. http:// www.ssk.de/SharedDocs/Beratungsergebnis-
se_PDF/2013/Ermittlung_Strahlenexposition. pdf?_blob=publicationFile. Zugegriffen: November 2016

35. Bundesamt für Strahlenschutz (2016) Hochspannungs-Gleichstrom-Übertragung (HGÜ). http:// www.bfs.de/DE/themen/emf/netzausbau/basiswissen/hgue/hgue_node.html. Zugegriffen: 18. März 2016

36. Sagunski H (1998) Richtwerte für die Innenraumluft: Styrol. Bundesgesundheitsblatt 41(1998):392421

37. Breuer K, Mayer F, Scherer C, Schwerd R, Sedlbauer K (2012) Wirkstoffauswaschung aus hydrophoben Fassadenbeschichtungen: verkapselte versus unverkapselte Biozidsysteme. Bauphysik 34(1):19-23

38. Leitfaden zur Vorbeugung, Untersuchung, Bewertung und Sanierung von Schimmelpilzwachstum in InnenräumenRatgeber: Schimmel im Haus. http://www.umweltbundesamt.de/sites/default/ files/medien/publikation/long/4218.pdf. Zugegriffen: November 2016

39. AWMF - das Portal der wissenschaftlichen Medizin: Schimmelpilzexposition in Innenräumen, medizinisch klinische Diagnostik; Registernummer 161 - 001 Klassifikation S2k Stand: 11. Apr. 2016 , gültig bis 10.04.2021. http://www.awmf.org/ uploads/tx_szleitlinien/161-001l_S2k_Schimmelpilzexposition-Innenraeume_2016-04.pdf. Zugegriffen: November 2016

40. Horvath I, Haas S, Knaller C, Sax G (2010) Health Impact Assessment.Konzept zur Etablierung von HIA in Österreich. Gesundheit Österreich. Bundesministeriums für Gesundheit, Wien

41. Amtsblatt EU (2009) Amtsblatt der Europäischen Union L53/41, 52. Jahrgang, 26. Februar 2009.

42. WHO (1999) Health 21 - Health for All in the 21th Century. European Health for All serie Bd. 6. WHO Regional Office for Europe, Copenhagen

43. Bundesministeriums der Justiz, für Verbraucherschutz (2010) BImSchV - Erste Verordnung zur Durchführung des Bundes-Immissionsschutzgesetzes (Verordnung über kleine und mittlere Feuerungsanlagen - 1. BImSchV. http://www.gesetzeim-internet.de/bundesrecht/bimschv_1_2010/ gesamt.pdf. Zugegriffen: 15. Febr. 2016

44. Branchenradar (2014) Dämmstoffe in Deutschland. http://www.marktmeinungmensch.de/ studien/branchenradar-daemmstoffe-in-deutschland-2014

45. EEG (2014) Gesetz für den Ausbau erneuerbarer Energien(Erneuerbare-Energien-Gesetz EEG 2014). https://www.bmwi.de/BMWi/Redaktion/PDF/G/ gesetz-fuer-den-ausbau-erneuerbarer-energien,pr operty=pdf,bereich=bmwi2012,sprache $=$ de,rwb= true.pdf. Zugegriffen: 15. Febr. 2016

46. IARC (2002) Non-lonizing Radiation Part 1: Staric and extremely low frequency electric and magnetic fields. IARC (International Agency for Research on Cancer) Monographs.

47. BMBF Forschungsvorhaben (2016) RiskAGuA Verbundprojekt: Risiken durch Abwässer aus der intensiven Tierhaltung für Grund und Oberflächenwasser. http://www.riskagua.de. Zugegriffen: 15. Febr. 2016

48. UBA-Ausschuss für Innenraumrichtwerte (1998) Richtwerte für die Innenraumluft: Styrol. Bundesgesundheitsblatt 41(9):392-421 